\title{
Editorial
}

\section{Recent Advances and Developments in Knee Surgery}

\author{
A. Malik ${ }^{1}$ and W.S. Khan ${ }^{*, 2, \S}$ \\ ${ }^{I}$ Department of Trauma and Orthopaedics, Queens Hospital, Barking Havering and Redbridge NHS Trust, Romford, \\ Essex, RM7 OAG, UK \\ ${ }^{2}$ University College London Institute of Orthopaedics and Musculoskeletal Sciences, Royal National Orthopaedic \\ Hospital, Stanmore, Middlesex, HA7 4LP, UK
}

In recent years there have been many advances and developments in all orthopaedic disciplines and knee surgery is no exception. These advances and developments in knee surgery have been due to our better understanding, and in turn better management of arthroses, fractures, and ligamentous injuries of the knee. These have been coupled with advances in our understanding of biomechanics as well as joint and ligament reconstruction, and arthroscopic surgery. All of these important developments are included in our special issue.

Isolated unicompartmental osteoarthritis in the young patient is a difficult problem to treat; they may be too young to consider total knee arthroplasty due to difficulties with inevitable future revision. Unicompartmental knee arthroplasty is one possible solution as it is perceived by some as being a smaller surgical insult than total knee arthroplasty, with easier revision to total knee arthroplasty than a revision total knee arthroplasty. A total knee arthroplasty performed as a revision unicondylar knee arthroplasty is thought by some authors to have equivalent functional outcomes to a primary total knee replacement. However, there have been several studies suggesting that revision is not as simple as suggested, and that function is not as good as primary total knee arthroplasty. In our series we include a systematic review of the literature regarding outcomes after revision of a unicondylar knee arthroplasty. Although there are many studies proposing selective use of the unicondylar knee arthroplasty there are a number of studies highlighting difficulties with revision and poorer outcomes, and, therefore, the unicondylar knee arthroplasty cannot be considered a small procedure that will 'buy time' for the patient, and have results equal to a primary knee arthroplasty when revised. Further controlled studies, ideally randomised, are required before final conclusions can be drawn.

\footnotetext{
*Address correspondence to this author at the University College London Institute of Orthopaedic and Musculoskeletal Sciences, Royal National Orthopaedic Hospital, Stanmore, Middlesex, HA7 4LP, UK; Tel: +44 (0) 7791 025554; Fax: + 44 (0) 7811 942192;

E-mail: wasimkhan@doctors.org.uk

${ }^{\S}$ Guest Editor
}

Total Knee Replacement is used to treat pain, stiffness and reduced range of movement. It has been estimated that a minimum of 90 degrees of range of motion in the knee is required for normal activities of daily living. In this series we include an article that demonstrates a technical note with a small patient series about the methods of treating knee stiffness after Total Knee Replacement.

Anterior Cruciate Ligament (ACL) rupture is a common sporting injury that frequently affects young, athletic patients. The graft choice for ACL reconstruction continues to be controversial. There are several options available for the treating surgeon, including Bone Patellar Tendon Bone (BPTB) grafts, Hamstring tendon (HT) grafts, allografts and synthetic grafts. Within the last decade there have been several comparative trials and meta-analysis, which have failed to provide an answer with regards to the best graft available. We look at the current concepts in graft choices for ACL reconstruction. ACL reconstruction has evolved significantly since the early 1900 's, back when an emphasis was placed on repair and not reconstruction. Over the past century, the technique has evolved from intra-articular non anatomic reconstruction, to extra articular reconstruction, back to intra articular (performed arthroscopically), to now, the advent of anatomic insertion site restoration. In this series, we illustrate the changes that have occurred, describing the rational for this process, based upon anatomical, radiological, biomechanical and clinical studies, all of which have aimed to improve patient function following ACL injury. Apart from the functional problems of instability, patients with ACL deficient knees also develop osteoarthritis. Although, this is frequently cited as an indication for ACL reconstruction, the relationship between $\mathrm{ACL}$ rupture, reconstruction and the instigation and progression of articular cartilage degenerative change is controversial. We review the published literature with regards ACL rupture and the multifactorial causes for osteoarthritis progression, and whether or not this is slowed or stopped by ACL reconstruction. There is no evidence in the published literature to support the view that ACL reconstruction prevents osteoarthritis, although it may prevent further meniscal damage.

The management of distal femoral, tibial and patellar fractures after total knee arthroplasty can be complex. The incidence of these fractures is increasing as the number of 
total knee arthroplasties being performed and patient longevity is increasing. There is a wide range of treatment options including revision arthroplasty for loose implants. We include a review article that discusses the epidemiology, risk factors, classification and treatment of these fractures.

High tibial osteotomy (HTO) is a procedure which aims to change the mechanical axis of the lower limb, transferring the body weight across healthy articular cartilage. Several studies have shown that accurate correction is the leading predictor for success. In this series we include a systematic review of the literature of computer-assisted techniques that have been used in attempts to increase the accuracy of the surgery and improve postoperative outcomes. The results of the cadaveric and clinical studies to date are presented and the benefits and pitfalls of navigation are discussed. We also include a systematic review to define the indications, functional outcomes, survivorship and complications associated with distal femoral varus osteotomy (DFVO). Cumulative survival with arthroplasty as the endpoint ranged from 64 to $82 \%$ at 10 years, and $45 \%$ at 15 years. The mean pre-operative HSS score ranged from 46 to 65 and this improved at latest follow up to means of between 72 and 88 . Pooled results show an overall complication rate of 5.8\% $(5 / 86)$ for unanticipated re-operation due to a complication. Poor reporting of included studies and considerable heterogeneity between them precluded any statistical analysis. Further study is required to determine the precise indications for DFVO, optimum surgical technique, implant of choice and post-operative rehabilitation regimen as all of these factors may significantly affect the complication profile and outcomes of this procedure. DFVO is technically demanding and requires a significant period of rehabilitation for the patient. However, long-term survivorship and good function have been demonstrated and it remains a potential option for valgus osteoarthritis in carefully selected patients.

Management of patellofemoral joint pathology is challenging as a result of the unique and complex organisation of static forces and dynamic factors contributing to its functional capacity. Anterior knee pain is a common presenting complaint, and in many cases no identifiable cause can be found. In these circumstances it is commonly known as anterior knee pain syndrome or patellofemoral pain syndrome. The management for this condition is most commonly non-operative. Treatment strategies include physiotherapy, pharmacotherapy, orthoses and combinations of the above. There are many described methods in the literature with a wide spectrum of outcomes, which in itself is testimony to the lack of any generally accepted gold standard of care for these patients. It is thus unclear to the health care professional treating these patients which is the best treatment to offer. We review the historical and most up to date literature on the subject and in so doing allow the health care professional pick whichever treatment strategy they feel most beneficial and also provide a guide for appropriate patient education.

Despite the complexity of the interplay of various components it is essential to attempt to describe patellar malalignement as a clinical entity in order to proceed with appropriate surgical management and successful outcomes. The goals of patellofemoral re- alignment surgery should be to create both a stable environment for optimal extensor mechanism performance and an appropriate load transmission for optimal cartilage wear and joint loading. In the context of this article we will review the operative management of patellofemoral malalignment; the indications for surgery, the different techniques available and the evidence regarding their effectiveness. A large number of procedures have been employed and they have all undergone various modifications over the course of the years. The majority of publications are retrospective series in poorly defined population groups. There are significant methodological inconsistencies and as a result there is lack of strong evidence base for the majority of these procedures. These are all described in a review in our series. Identification and management of patients with isolated patellofemoral osteoarthritis is challenging. Many of these patients present at a young age and it is important to distinguish degenerative change in the patellofemoral articulation from the other various causes of anterior knee pain. Once the diagnosis of isolated patellofemoral arthrosis has been made non-operative and conservative surgical techniques should be exhausted fully before prosthetic arthroplasty is considered. We include a systematic review of the literature that focuses on the use of arthroplasty for isolated patellofemoral arthrosis, in particular comparing the use of total knee against selective patellofemoral joint replacements.

The menisci disperse the load at the knee joint. Removal of the menisci can lead to osteoarthritis due to the higher load placed on the underlying cartilage. If they become injured it is therefore important to replace or regenerate the meniscus to prevent the progression of osteoarthritis. Many materials have been trialled to find a scaffold that can withstand the stresses and strains across the joint without causing any adverse effects. Lastly we include a review that looks at these materials further to clarify the current position of tissue engineering for the meniscus and to highlight the areas where further research is needed. A scaffold which can produce high quality in vivo results in everyone has not yet been found.

We hope that all surgeons involved in the management of knee pathologies find this issue useful.

(C) Malik and Khan; Licensee Bentham Open.

This is an open access article licensed under the terms of the Creative Commons Attribution Non-Commercial License (http://creativecommons.org/licenses/by-nc/3.0/) which permits unrestricted, non-commercial use, distribution and reproduction in any medium, provided the work is properly cited. 